EL EFECTO DEL DESARROLLO DE PLANES DE NEGOCIO EN LAS ACTITUDES E INTENCIONES EMPRENDEDORAS DE LOS ESTUDIANTES.

\title{
EL EFECTO DEL DESARROLLO DE PLANES DE NEGOCIO EN LAS ACTITUDES E INTENCIONES EMPRENDEDORAS DE LOS ESTUDIANTES
}

\section{THE EFFECT OF DEVELOPING BUSINESS PLANS ON STUDENTS' ENTREPRENEURIAL ATTITUDES AND INTENTIONS}

Omar Heredia Portillo*, Luz Amparo Delgado Díaz**, Elba Martina Cortés Palacios ${ }^{\star \star \star}$

\footnotetext{
* Universidad de Guadalajara, Centro Universitario de la Costa, Av. Universidad, Delegación Ixtapa, Puerto Vallarta, Jal., C.P. 48290, México. ORCID: http://orcid.org/0000-0002-2239-996X.

** Universidad de Guadalajara, Centro Universitario de la Costa, Av. Universidad, Delegación Ixtapa, Puerto Vallarta, Jal., C.P. 48290, México. Email: jab1298@gmail.com, ORCID: http://orcid.org/0000-0003-23884737.

*** Universidad de Guadalajara, Centro Universitario de la Costa, Av. Universidad, Delegación Ixtapa, Puerto Vallarta, Jal., C.P. 48290, México. Email: cortes_elba@hotmail.com, ORCID: http://orcid.org/00000002-1988-8068.
}

Dirección para recibir correspondencia: omar.heredia@alumno.udg.mx 
EL EFECTO DEL DESARROLLO DE PLANES DE NEGOCIO EN LAS ACTITUDES E INTENCIONES EMPRENDEDORAS DE LOS ESTUDIANTES.

\section{RESUMEN}

OBJETIVO: Analizar el efecto del desarrollo de planes de negocio en las actitudes e intenciones emprendedoras de los estudiantes aplicando la teoría de la conducta planeada.

MATERIAL Y MÉTODO: Mediante un diseño de estudio transversal se comparó un grupo de tratamiento y un grupo comparativo para determinar si hubo diferencias estadísticamente significativas en las actitudes e intenciones emprendedoras después de desarrollar un plan de negocio.

RESULTADOS: Desarrollar planes de negocio no tiene efecto en las actitudes hacia el emprendimiento, normas subjetivas e intenciones emprendedoras, pero si tiene efecto en el control conductual percibido.

CONCLUSIONES: Escribir planes de negocio en un programa de desarrollo de emprendedores no es suficiente para que los estudiantes pasen de la intención a la acción de crear un nuevo negocio. Es necesario buscar más herramientas para reforzar la educación emprendedora.

Palabras clave: Intenciones emprendedoras. Actitudes emprendedoras. Desarrollo de planes de negocio. Educación emprendedora. Teoría de la conducta planeada.

\section{ABSTRACT}

OBJECTIVE: To analyze the effect of the development of business plans on the entrepreneurial attitudes and intentions of students applying the theory of planned behavior.

MATERIAL AND METHOD: Using a cross-sectional study design, a treatment group and a comparative group were compared to determine if there were statistically significant differences in entrepreneurial attitudes and intentions after developing a business plan.

RESULTS: Developing business plans has no effect on attitudes towards entrepreneurship, subjective norms, and entrepreneurial intentions, but it does on perceived behavioral control. 
EL EFECTO DEL DESARROLLO DE PLANES DE NEGOCIO EN LAS ACTITUDES E INTENCIONES EMPRENDEDORAS DE LOS ESTUDIANTES.

CONCLUSIONS: Writing business plans in an entrepreneurial development program is not enough to move students from intention to action on creating a new business. It is necessary to look for more tools that strengthen entrepreneurial education.

KEYWORDS: Entrepreneurial intentions. Entrepreneurial attitudes. Development of business plans. Entrepreneurial education. Theory of planned behavior.

\section{INTRODUCCIÓN}

En días recientes, los educadores y profesionales en negocios tienen claro que el emprendimiento, o ciertas facetas de este pueden ser enseñadas (Kuratko, 2005). De esta forma, las diferentes instituciones educativas comienzan a reconocer la importancia del emprendimiento en la educación introduciendo cursos específicos sobre la materia (Aldana, Ibarra, \& Lowenstein, 2011). Según, Blank et al. (2014), la educación emprendedora tiene sus orígenes en las escuelas de negocios que surgen por la necesidad de los corporativos en Estados Unidos (EU) de una clase profesional de dirección. Su auge comenzó en 1971 (Katz, 2003), y para 1984 se introdujeron las primeras competencias en planes de negocios en las principales universidades de EU, que más tarde se convirtieron en la materia más importante en los cursos de emprendimiento (Honig, 2012; Karlsson, 2005).

Sin embargo, aunque el formato del plan de negocio (BP, por sus siglas en inglés) puede ser conveniente, no hay evidencia de que ayude a los estudiantes a aprender los aspectos necesarios del campo (Furr, 2012; Bewayo, 2015; Honig, 2004). El asunto es que en la ausencia de un proceso alternativo que guíe un emprendimiento, los emprendedores recurren a la planeación y ejecución creyendo que si sólo escriben un BP o desarrollan un producto tendrán éxito, pero el resultado es el fracaso (Ahlstrom \& Furr, 2011). En consecuencia, esta investigación evalúa el efecto del desarrollo de BP en las actitudes e intenciones emprendedoras de los estudiantes. La teoría de la conducta planeada (TPB, por sus siglas en inglés) de Ajzen (1991) ofreció un marco teórico adecuado (Krueger \& Carsrud, 1993; Fayolle \& Degeorge, 2006). Para aumentar la fiabilidad en la medición del efecto se incluyó un grupo de tratamiento y un grupo comparativo (Tung, 2011; Karali, 2013; Fayolle \& Liñán, 2014). Los resultados muestran que la TPB ofrece una estructura teórica adecuada para evaluar el efecto de la educación emprendedora en los 
EL EFECTO DEL DESARROLLO DE PLANES DE NEGOCIO EN LAS ACTITUDES E INTENCIONES EMPRENDEDORAS DE LOS ESTUDIANTES.

estudiantes, y el desarrollo de BP no es suficiente para reforzar la decisión de los estudiantes en convertirse en emprendedores.

\section{Revisión de la literatura}

\section{Teoría de la conducta planeada}

La TPB es apropiada para analizar cómo y en qué condiciones el proceso de desarrollar un BP dentro de un programa de educación afecta las intenciones emprendedoras (Autio, Keeley, Klofsten, Parker, \& Hay, 2001; Díaz-García, Sáez-Martínez, \& Jiménez-Moreno, 2015; Fayolle \& Degeorge, 2006; Karali, 2013; Krueger \& Carsrud, 1993; Liñán , 2004; Liñán \& Chen, 2009; Tung, 2011; Qureshi \& Mahdi, 2014). Ajzen (1991) dice que, el factor central en la TPB es la intención del individuo para desempeñar una conducta dada, cual captura los factores motivacionales que la influyen; e indica la fuerza de disposición de la persona a intentar, o cuanto esfuerzo está planeando ejercer para desempeñar la conducta, por lo que, entre más fuerte es la intención mayor probabilidad existe de realizarse. TPB permite estudiar y entender las diferentes actitudes que sostienen la intención emprendedora y examinar los antecedentes que influyen tales actitudes (Lima, Nassif, Lopes, \& Silva, 2014). Ajzen (1991) comenta que, los determinantes de la intención son tres: (1) actitud hacia la conducta, que es el grado en que una persona tiene una evaluación o valoración favorable o desfavorable de la conducta en cuestión; (2) normas subjetivas, que es la percepción de presión social para desempeñar o no la conducta y; (3) percepción de control de la conducta, que es la percepción de facilidad o dificultad de desempeñar la conducta y que su supone reflejar experiencias pasadas, al igual que los obstáculos e impedimentos anticipados.

De acuerdo con la TPB la intención preside la acción (Lima, Nassif, Lopes, \& Silva, 2014), en este sentido, Shook, Priem, \& McGee (2003) argumentan que el proceso de creación de empresas inicia cuando un individuo desarrolla la intención; una vez que la intención está formada, comienza la búsqueda y descubrimiento de las oportunidades; posteriormente, la decisión de explotarlas; y finalmente, la acción traduce el intento en una nueva empresa. Por lo que se establecen las siguientes hipótesis de asociación: (H1) La actitud hacia el emprendimiento está positivamente relacionada con la intención emprendedora; (H2) Las normas subjetivas están positivamente relacionadas con la intención emprendedora; $(\mathrm{H} 3)$ La percepción de control conductual está positivamente relacionado con la intención emprendedora. 
EL EFECTO DEL DESARROLLO DE PLANES DE NEGOCIO EN LAS ACTITUDES E INTENCIONES EMPRENDEDORAS DE LOS ESTUDIANTES.

\section{Desarrollo de planes de negocio en la educacion emprendedora}

Los BPs son la parte central de los cursos académicos básicos sobre emprendimiento (Bewayo, 2015; Lange et al., 2011). Muchos educadores creen que escribir un BP afecta la autoeficacia, incrementa la intención emprendedora y la probabilidad de comenzar realmente un negocio más que cualquier otra pedagogía (Lange, Marram, Jawahar, Yong, \& Bygrave, 2011). Hormozi et al. (2002), sugiere que el BP juega un rol importante en determinar el grado de éxito esperado por un emprendedor porque le ayuda a clarificar sus objetivos a corto y largo plazo. Según Cook, Belliveau, \& Lentz (2007) a mayor habilidad para desarrollarlos el emprendedor estará mejor equipado para encontrar inversión y reforzar el potencial de crecimiento de la nueva empresa.

Cook, Belliveau, \& Sandberg (2004) consideran que el desarrollo de un BP de calidad es un ingrediente esencial para el éxito emprendedor. Longenecker et al. (2010) sostienen que, son un requisito previo que ayuda al emprendedor a comunicar su visión y enfocarse en los aspectos y las actividades más importantes, y juegan un papel crucial en la evaluación global de una nueva empresa por parte de inversionistas, aumentando las posibilidades de éxito (Ashamalla, Orife, \& Abel, 2008). Por lo tanto, los estudiantes que han aprendido a desarrollar BPs deben mostrar un mayor conocimiento y habilidades emprendedoras, autoeficacia e intención de iniciar una nueva empresa (Lange, Marram, Jawahar, Yong, \& Bygrave, 2011; Honig, 2004). Sin embargo, aunque desarrollar un BP es el tema y método de enseñanza más popular en los cursos de emprendimiento ofrecidos por colegios y universidades, no hay una evidencia sólida de su importancia para el éxito del emprendedor (Furr, 2012; Honig, 2004; Solomon, 2007). Por el contrario, una creciente evidencia sugiere que, a pesar del gran énfasis por los académicos, este no se relaciona con el éxito, pudiendo ser una pérdida de tiempo (Furr, 2012; Eschker, Gold, \& Lane, 2017).

De acuerdo con Lange et al. (2007), no hay diferencias en el desempeño de las nuevas empresas que inician con o sin un BP, y a menos que un emprendedor necesite financiamiento de alguna institución no existe otra razón para escribirlos antes de emprender. Según Bhidé (1999) una vez que el emprendedor encuentra la idea correcta no gasta mucho tiempo en investigación y planeación. Además, la correlación del BP de una empresa de reciente creación y su supervivencia se ha encontrado ser muy débil (Bewayo, 2015). Incluso, no se ha probado si realmente ayuda a los emprendedores a tomar mejores decisiones (Honig, 2004). La modalidad de enseñanza del emprendimiento limitada al desarrollo de BP no incrementa el deseo e interés HEREDIA-PORTILLO O., DELGADO-DÍAZ L. A., CORTÉS-PALACIOS E. M. 
EL EFECTO DEL DESARROLLO DE PLANES DE NEGOCIO EN LAS ACTITUDES E INTENCIONES EMPRENDEDORAS DE LOS ESTUDIANTES.

de ser emprendedor en los estudiantes (Tang, Chen, Li, \& Lu, 2014). Por lo tanto, no debería ser la actividad focal de aprendizaje en este tipo de educación (Colin \& Andy, 2013; Furr, 2012).

En consecuencia, la educación emprendedora basada en el desarrollo de BP, trae como resultado que los estudiantes no obtengan la experiencia directa en emprendimiento que necesitan para pasar de una intención a la acción de crear una nueva empresa (Díaz-García, Sáez-Martínez, \& Jiménez-Moreno, 2015); no desarrollen competencias emprendedoras, sino más bien desarrollen habilidades gerenciales (Aldana, Ibarra, \& Lowenstein, 2011); por lo tanto, no estén preparados para la construcción de nuevos negocios (Blank, Engel, \& Hornthal, 2014). Esta utilidad cuestionable de los BPs, lleva a suponer que cualquier clase de planeación para iniciar un negocio podría no ser útil, y que su desarrollo formal no se requiere en un curso de educación emprendedora (Vincett \& Farlow, 2008). Honig \& Samuelsson (2011) comentan que, el esfuerzo para enseñar planeación a nivel universitario podría ser en realidad una mala asignación de recursos pedagógicos. Esto apunta a la necesidad de revisar el énfasis de la educación emprendedora en el desarrollo de BPs, mediante el análisis de su efecto en las actitudes e intenciones emprendedoras (Lange, Mollov, Pearlmutter, Singh, \& Bygrave, 2007). En este punto, se plantean las siguientes hipótesis de diferencia de grupos: (H4) No existen diferencias significativas en la actitud hacia el emprendimiento entre los estudiantes expuesto al desarrollo del plan de negocio y los no expuestos; (H5) No existen diferencias significativas en las normas subjetivas entre los estudiantes expuesto al desarrollo del plan de negocio y los no expuestos; (H6) No existen diferencias significativas en el control conductual percibido entre los estudiantes expuesto al desarrollo del plan de negocio y los no expuestos; (H7) No existen diferencias significativas en la intención emprendedora entre los estudiantes expuesto al desarrollo del plan de negocio y los no expuestos.

\section{MATERIAL Y MÉTODO}

\section{Modelo teórico}

Acorde con la teoría de la conducta planeada, la intención emprendedora indica el esfuerzo que la persona hará para llevar a cabo esa conducta emprendedora (Liñán \& Chen, 2009). Por lo tanto, esto captura los tres factores motivacionales que influyen la conducta: actitud hacia el emprendimiento, normas subjetivas y control conductual percibido (Ajzen, 1991; Liñán, 2004). Por otro lado, el desarrollo de planes de negocio es la parte central de los cursos básicos sobre 
EL EFECTO DEL DESARROLLO DE PLANES DE NEGOCIO EN LAS ACTITUDES E INTENCIONES EMPRENDEDORAS DE LOS ESTUDIANTES.

emprendimiento y el principal instrumento pedagógico de la educación emprendedora (Aldana, Ibarra, \& Lowenstein, 2011; Blank, Engel, Hornthal, 2014; Katz, 2003; Honig, 2004; Solomon, 2007). Basado en lo anterior, el desarrollo de planes de negocio debería afectar las actitudes e intenciones emprendedoras de los participantes; sin embargo, la revisión teórica indica lo contrario.

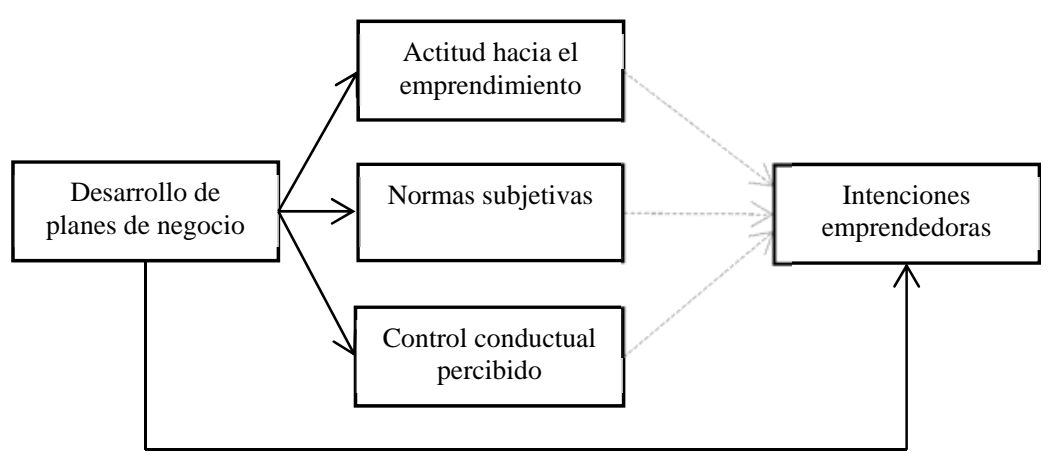

Figura 1. Modelo teórico.

Fuente: Elaboración propia con base en Ajzen (1991) y Fayolle \& Degeorge (2006).

\section{Conceptualización y operacionalización de las variables}

Variables dependientes: (1) intenciones emprendedoras, son el grado de convicción percibida por el individuo de intentar establecer un nuevo negocio y, que conscientemente planea hacerlo en algún momento en el futuro (Thompson, 2009); (2) actitud hacia el emprendimiento, es el grado en que el individuo tiene una valoración personal positiva o negativa de ser un emprendedor (Ajzen, 2001; Autio, Keeley, Klofsten, Parker, \& Hay, 2001): (3) normas subjetivas, son el grado de presión social percibida para llevar o no a cabo un emprendimiento (Liñán \& Chen, 2009). En particular, se refiere a la percepción de lo que las personas importantes en la vida del individuo piensan acerca de su decisión de convertirse en emprendedor (Karali, 2013); y (4) control conductual percibido, es el grado de percepción de la facilidad o dificultad de convertirse en emprendedor (Liñán, 2004), y la percepción de confianza en las habilidades personales para tener éxito (Karali, 2013).

Variable independiente: desarrollo de planes de negocio, es la exposición a la herramienta pedagógica de la educación emprendedora (Aldana, Ibarra, \& Lowenstein, 2011; Blank, Engel \& Hornthal, 2014; Katz, 2003; Honig, 2004). Medida objetivamente cuando los encuestados HEREDIA-PORTILLO O., DELGADO-DÍAZ L. A., CORTÉS-PALACIOS E. M.

MAYO-AGOSTO 2020. Año 26, Número 75. Págs. 157-175 
EL EFECTO DEL DESARROLLO DE PLANES DE NEGOCIO EN LAS ACTITUDES E INTENCIONES EMPRENDEDORAS DE LOS ESTUDIANTES.

respondieron haber desarrollado o no un plan de negocio en el curso de desarrollo de emprendedores (Honig \& Samuelsson, 2011; Burke, Fraser, \& Greene, 2010; Brinckmann, Grichnik, \& Kapsa, 2010). Variables de control: se controló el género, la edad, la carrera profesional y la experiencia laboral debido a que estudios previos han indicado que pueden afectar las actitudes e intenciones de iniciar un negocio (Kolvereid \& Moen, 1997; Morris \& Venkatesh, 2000; Lévesque \& Minniti, 2006; Colombatto \& Melnik, 2007).

\section{Diseño y participantes}

La población consistió en alumnos registrados en el curso de desarrollo de emprendedores y alumnos estudiando un semestre previo al ingreso al curso de las carreras en administración, contabilidad y turismo del Centro Universitario de la Costa (Cucosta) de la Universidad de Guadalajara en el ciclo escolar 2016A, con base al Sistema Integral de Información y Administración Universitaria (SIIAU) consultado el 25 de enero de 2016. Dos muestras de 117 estudiantes cada una, en total 234 estudiantes, fue obtenida mediante un método de muestreo por cuotas (Hernández, Fernández, \& Baptista, 2014; García Cabrero, 2009), y se fijaron las proporciones siguientes: administración 38\%, contabilidad 45\%, y turismo $17 \%$. El diseño de la investigación fue transversal correlacional por ser considerado fiable para investigar las intenciones emprendedoras (Autio, Keeley, Klofsten, Parker, \& Hay, 2001; Krueger \& Reilly, 2000; Karali, 2013), las mediciones fueron hechas en un momento en el tiempo (García Cabrero, 2009), y por qué se buscó establecer relaciones estadísticas entre algunas de las variables y probar hipótesis (Tamayo y Tamayo, 2012; García Cabrero, 2009; Hernández, Fernández, \& Baptista, 2014). El grupo comparativo o de control tuvo el objetivo de aumentar la fiabilidad en la medición del efecto del desarrollo del plan de negocio en los participantes (Tung, 2011; Fayolle \& Liñán, 2014). La encuesta de Liñán \& Chen (2009) fue la técnica de recolección de datos. Y para medir la intensidad de las actitudes e intenciones de una forma objetiva se usó un instrumento con escala de actitudes tipo Likert de siete afirmaciones de 1= totalmente en desacuerdo a $7=$ totalmente de acuerdo (García Cabrero, 2009; Tamayo y Tamayo, 2012). La confiabilidad el instrumento se determinó por un coeficiente de Alpha de Cronbach $>.70$. Esto fue el resultado de una prueba piloto, que permitió modificar, ajustar y mejorar los indicadores de confiabilidad y validez (García Cabrero, 2009). 
EL EFECTO DEL DESARROLLO DE PLANES DE NEGOCIO EN LAS ACTITUDES E INTENCIONES EMPRENDEDORAS DE LOS ESTUDIANTES.

\section{Procedimiento}

En la investigación, se eligieron dos grupos, uno inscrito por primera vez a la materia de desarrollo de emprendedores (de aquí en adelante, expuestos al desarrollo del plan de negocio) y uno no inscrito a la materia (grupo de control), siguiendo la recomendación de Fayolle \& Liñán (2014). Los grupos elegidos mostraron ser homogéneos en la carrera, sexo, rango de edad y experiencia laboral. Su única diferencia fue la presencia o ausencia en la exposición al desarrollo de un plan de negocio, lo que hizo viable su comparación para analizar el efecto del plan de negocio sobre las actitudes e intenciones emprendedoras (Tung, 2011; Fayolle \& Liñán, 2014). Al finalizar el curso de desarrollo de emprendedores la encuesta fue aplicada a ambos grupos en el salón de clases con el permiso de las autoridades académicas correspondientes. La única diferencia en la encuesta fue la instrucción para el grupo expuesto, a ellos se les preguntó su percepción acerca de la utilidad del desarrollo del plan de negocio para reforzar las actitudes e intenciones emprendedoras.

Para reducir el sesgo en reportar la percepción acerca de las variables, las instrucciones de la encuesta enfatizaron la importancia de la honestidad al contestar, además, se hizo hincapié en la confidencialidad con base en la ley de protección a los datos personales, incluso se puntualizó la inexistencia de respuestas correctas y la autonomía de los resultados al desempeño académico de los participantes. También, se pidió a los encuestados responder con base a su percepción acerca de la pregunta, sin dejar respuestas en blanco. Al término de la recopilación de los datos se procedió a su captura y análisis mediante el programa SPSS (Paquete Estadístico Aplicado a las Ciencias Sociales en su versión para Windows). El primer paso fue explorar los datos obtenidos mediante histogramas y boxplot y las pruebas de Kolmogorov-Smirnov. Posteriormente, se investigó de manera objetiva la fuerza y dirección de la relación entre las variables del modelo de intención a través del coeficiente de correlación de Spearman. Finalmente, se analizó el efecto del desarrollo del plan de negocio mediante la comparación de las actitudes e intenciones emprendedoras entre el grupo expuesto y el grupo de control usando la prueba $U$ de Mann-Whitney.

La exposición al desarrollo de un plan de negocio, consistió en tomar un curso de desarrollo de emprendedores ofrecido por el departamento de ciencias económico-administrativas a los alumnos de las carreras en administración, contabilidad y turismo, en el ciclo 2016 A del Centro Universitario de la Costa de la Universidad de Guadalajara. El curso estuvo integrado por 40 HEREDIA-PORTILLO O., DELGADO-DÍAZ L. A., CORTÉS-PALACIOS E. M. 
EL EFECTO DEL DESARROLLO DE PLANES DE NEGOCIO EN LAS ACTITUDES E INTENCIONES EMPRENDEDORAS DE LOS ESTUDIANTES.

horas teóricas y 40 horas prácticas. Las actividades prácticas y la evaluación del programa se evidenciaron mediante el desarrollo de las etapas de un plan de negocio (prototipo de idea de negocio, plan de mercadotecnia, plan técnico y plan financiero) y la presentación de este al final del curso (UDG, 2016a). Este curso emprendedor es el único ofrecido por el departamento de ciencias económico-administrativas, con el objetivo de desarrollar las capacidades y habilidades que los estudiantes necesitan para realizar actividades emprendedoras.

\section{RESULTADOS}

Para conocer la distribución de los datos se realizó la prueba de Kolmogorov-Smirnov (García Cabrero, 2009). De acuerdo con Hinton et al. (2004), los valores deben ser $p>0.05$ para indicar que la distribución observada es una distribución normal. La tabla 1, muestra que todas las variables tienen un nivel de significancia $p<0.05$; es decir, se asume que no están normalmente distribuidas.

Tabla 1

Pruebas de normalidad de los grupos participantes

\begin{tabular}{llccc}
\hline & & \multicolumn{3}{c}{ Kolmogorov-Smirnov $^{\text {a }}$} \\
\hline \multirow{2}{*}{ Actitud al Emprendimiento } & Grupo & Estadístico & gl & Sig. \\
& Expuesto & .237 & 117 & .000 \\
Control Conductual & Control & .234 & 117 & .000 \\
& Expuesto & .092 & 117 & .016 \\
Normas Subjetivas & Control & .123 & 117 & .000 \\
& Expuesto & .215 & 117 & .000 \\
Intención Emprendedora & Control & .170 & 117 & .000 \\
& Expuesto & .188 & 117 & .000 \\
\hline
\end{tabular}

a. Corrección de la significación de Lilliefors.

Fuente: Elaboración propia con base en datos empíricos de estudio de campo.

Debido a los resultados de la prueba de normalidad, se justifica el uso de pruebas no paramétricas para probar las hipótesis de esta investigación (García Cabrero, 2009; Liñán \& Chen, 2009). Por lo tanto, para medir el nivel de asociación se utilizó el coeficiente de correlación de Spearman. Los resultados de la tabla 2 , muestran que existe una relación positiva moderada $\left(\mathrm{r}_{\mathrm{s}}=0.669, \mathrm{p}<\right.$ 0.01 ) entre la actitud al emprendimiento y la intención emprendedora, soportando H1. En relación con las normas subjetivas, esta presenta una asociación positiva débil $\left(r_{s}=0.361, p<0.01\right)$, pero significativa con la intención emprendedora, soportando $\mathrm{H} 2$. Finalmente, los resultados muestran HEREDIA-PORTILLO O., DELGADO-DÍAZ L. A., CORTÉS-PALACIOS E. M. 
EL EFECTO DEL DESARROLLO DE PLANES DE NEGOCIO EN LAS ACTITUDES E INTENCIONES EMPRENDEDORAS DE LOS ESTUDIANTES.

que el control conductual percibido tiene una asociación moderada $\left(r_{s}=0.508, p<0.01\right)$ con la intención emprendedora, soportando H3.

Tabla 2

Rho de Spearman para el grupo expuesto

\begin{tabular}{|c|c|c|c|c|c|c|}
\hline & & & $\begin{array}{l}\text { Intención de } \\
\text { Emprender }\end{array}$ & $\begin{array}{c}\text { Actitud al } \\
\text { Emprendimiento }\end{array}$ & $\begin{array}{c}\text { Control } \\
\text { Conductual }\end{array}$ & $\begin{array}{l}\text { Normas } \\
\text { Subjetivas }\end{array}$ \\
\hline \multirow{12}{*}{$\begin{array}{l}\text { Rho de } \\
\text { Spearman }\end{array}$} & \multirow{3}{*}{$\begin{array}{l}\text { Intención } \\
\text { Emprendedora }\end{array}$} & Coeficiente de correlación & 1.000 & $.669^{* *}$ & $.508^{* *}$ & $.361^{* *}$ \\
\hline & & Sig. (bilateral) & 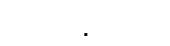 & .000 & .000 & .000 \\
\hline & & $\mathrm{N}$ & 117 & 117 & 117 & 117 \\
\hline & \multirow{3}{*}{$\begin{array}{l}\text { Actitud al } \\
\text { Emprendimiento }\end{array}$} & Coeficiente de correlación & $.669^{* *}$ & 1.000 & $.533^{* *}$ & $.472^{* *}$ \\
\hline & & Sig. (bilateral) & .000 & & .000 & .000 \\
\hline & & $\mathrm{N}$ & 117 & 117 & 117 & 117 \\
\hline & Control & Coeficiente de correlación & $.508^{* *}$ & $.533^{* \star}$ & 1.000 & $.443^{\star *}$ \\
\hline & \multirow{2}{*}{ Conductual } & Sig. (bilateral) & .000 & .000 & . & .000 \\
\hline & & $\mathrm{N}$ & 117 & 117 & 117 & 117 \\
\hline & Normas & Coeficiente de correlación & $.361^{\star *}$ & $.472^{* *}$ & $.443^{* *}$ & 1.000 \\
\hline & \multirow{2}{*}{ Subjetivas } & Sig. (bilateral) & .000 & .000 & .000 & . \\
\hline & & $\mathrm{N}$ & 117 & 117 & 117 & 117 \\
\hline
\end{tabular}

**. La correlación es significativa al nivel 0,01 (bilateral).

Fuente: Elaboración propia con base en datos empíricos de estudio de campo.

Debido a la distribución de los datos, la prueba $U$ de Mann-Whitney es la alternativa no paramétrica de la t de Student para muestras independientes (García Cabrero, 2009). El objetivo es probar que las dos muestras han sido tomadas de la misma población. Sin embargo, una de las muestras sufrió los efectos de la variable independiente (desarrollo de un plan de negocio), por lo que se esperan diferencias. Como ambas muestras son grandes es conveniente reportar el valor de (Z) (Levin \& Rubin 2004; Hinton, Brownlow, McMurray, \& Cozens, 2004). En consecuencia, para un nivel de significancia de 0.05 el valor critico de (Z) es de 1.96 (Levin \& Rubin, 2004). La tabla 3 , muestra que a pesar de que el rango promedio de la actitud hacia el emprendimiento del grupo del grupo expuesto (113.81) es menor que el del grupo de control (121.19) la diferencia no es estadísticamente significativa $(Z=0.848, p>0.05)$, soportando $\mathrm{H} 4$.

Tabla 3

Rangos y estadísticos de contraste de la actitud hacia el emprendimiento

\begin{tabular}{lccccccc}
\hline Muestra & $\mathrm{N}$ & $\begin{array}{c}\text { Rango } \\
\text { promedio }\end{array}$ & $\begin{array}{c}\text { Suma de } \\
\text { rangos }\end{array}$ & $\begin{array}{c}\text { Ude Mann- } \\
\text { Whitney }\end{array}$ & $\begin{array}{c}\text { W de } \\
\text { Wilcoxon }\end{array}$ & $\begin{array}{c}\text { Z } \\
\text { Sig. asintót. } \\
\text { (bilateral) }\end{array}$ \\
\hline Expuestos & 117 & 113.81 & 13315.50 & 6412.500 & 13315.500 & -.848 & .397 \\
Control & 117 & 121.19 & 14179.50 & & & & \\
Total & 234 & & & & & & \\
\hline
\end{tabular}

Fuente: Elaboración propia con base en datos empíricos de estudio de campo. 
EL EFECTO DEL DESARROLLO DE PLANES DE NEGOCIO EN LAS ACTITUDES E INTENCIONES EMPRENDEDORAS DE LOS ESTUDIANTES.

Aunque el rango promedio de las normas subjetivas del grupo expuesto (119.73) es mayor que el del grupo de control (115.27) la diferencia no es estadísticamente significativa ( $Z=0.521$, p>0.05), soportando H5 (ver tabla 4).

Tabla 4

Rangos y estadísticos de contraste de las normas subjetivas

\begin{tabular}{lccccccc}
\hline Muestra & $\mathrm{N}$ & $\begin{array}{c}\text { Rango } \\
\text { promedio }\end{array}$ & $\begin{array}{c}\text { Suma de } \\
\text { rangos }\end{array}$ & $\begin{array}{c}\text { Ude Mann- } \\
\text { Whitney }\end{array}$ & $\begin{array}{c}\text { W de } \\
\text { Wilcoxon }\end{array}$ & $\begin{array}{c}\text { Z } \\
\text { Sig. asintót. } \\
\text { (bilateral) }\end{array}$ \\
\hline Expuestos & 117 & 119.73 & 14008.50 & 6583.500 & 13486.500 & -.521 & .602 \\
Control & 117 & 115.27 & 13486.50 & & & & \\
Total & 234 & & & & & & \\
\hline
\end{tabular}

Fuente: Elaboración propia con base en datos empíricos de estudio de campo.

Como muestra la tabla 5, el rango promedio del control conductual percibido del grupo expuesto (127.20) es mayor que el del grupo de control (107.80). Esta diferencia es estadísticamente significativa $(Z=2.199, \mathrm{p}<0.05)$, lo cual no soporta $\mathrm{H} 6$.

\section{Tabla 5}

Rangos y estadísticos de contraste del control conductual percibido

\begin{tabular}{lccccccc}
\hline Muestra & $\mathrm{N}$ & $\begin{array}{c}\text { Rango } \\
\text { promedio }\end{array}$ & $\begin{array}{c}\text { Suma de } \\
\text { rangos }\end{array}$ & $\begin{array}{c}\text { Ude Mann- } \\
\text { Whitney }\end{array}$ & $\begin{array}{c}\text { W de } \\
\text { Wilcoxon }\end{array}$ & $\begin{array}{c}\text { Z } \\
\text { Sig. asintót. } \\
\text { (bilateral) }\end{array}$ \\
\hline $\begin{array}{lcccccc}\text { Expuestos } \\
\text { Control }\end{array}$ & 117 & 127.20 & 14882.50 & 5709.500 & 12612.500 & -2.199 & .028 \\
Total & 117 & 107.80 & 12612.50 & & & & \\
\hline
\end{tabular}

Fuente: Elaboración propia con base en datos empíricos de estudio de campo.

La tabla 6, muestra que a pesar de que el rango promedio de la intención emprendedora del grupo expuesto (116.07) es menor que el del grupo de control (118.93) la diferencia no es estadísticamente significativa $(Z=0.327, \mathrm{p}>0.05)$, soportando $\mathrm{H} 7$.

Tabla 6

Rangos y estadísticos de contraste de la intención emprendedora

\begin{tabular}{lccccccc}
\hline Muestra & $\mathrm{N}$ & $\begin{array}{c}\text { Rango } \\
\text { promedio }\end{array}$ & $\begin{array}{c}\text { Suma de } \\
\text { rangos }\end{array}$ & $\begin{array}{c}\text { U de Mann- } \\
\text { Whitney }\end{array}$ & $\begin{array}{c}\text { W de } \\
\text { Wilcoxon }\end{array}$ & $\begin{array}{c}\text { Z } \\
\text { Sig. asintót. } \\
\text { (bilateral) }\end{array}$ \\
\hline Expuestos & 117 & 116.07 & 13580.00 & 6677.000 & 13580.000 & -.327 & .743 \\
Control & 117 & 118.93 & 13915.00 & & & & \\
Total & 234 & & & & & \\
\hline
\end{tabular}

Fuente: Elaboración propia con base en datos empíricos de estudio de campo. 
EL EFECTO DEL DESARROLLO DE PLANES DE NEGOCIO EN LAS ACTITUDES E INTENCIONES EMPRENDEDORAS DE LOS ESTUDIANTES.

\section{CONCLUSIONES}

El propósito de este artículo fue analizar el efecto del desarrollo del plan de negocio en las actitudes e intenciones emprendedoras de los estudiantes aplicando la teoría de la conducta planeada, debido a que muchos estudios empíricos han demostrado su utilidad en el estudio del efecto de la educación emprendedora en los participantes (Autio, Keeley, Klofsten, Parker, \& Hay, 2001; Díaz-García, Sáez-Martínez, \& Jiménez-Moreno, 2015; Fayolle \& Degeorge, 2006; Karali, 2013; Krueger \& Carsrud, 1993; Liñán , 2004; Liñán \& Chen, 2009; Tung, 2011; Qureshi \& Mahdi, 2014). Para medir el efecto del desarrollo del plan de negocio en las actitudes e intenciones se usó el cuestionario propuesto por Liñán \& Chen (2009) y, como sugieren Tung (2011) y Fayolle \& Liñán (2014) se incluyó un grupo de tratamiento (estudiantes que desarrollaron un plan de negocio) y un grupo comparativo (estudiantes que no desarrollaron un plan de negocio) para aumentar la fiabilidad.

En concordancia con Fayolle \& Degeorge (2006) y Krueger \& Carsrud (1993), los resultados revelan que la TPB es apropiada para abordar el fenómeno. La actitud hacia el emprendimiento $\left(r_{s}=0.669\right)$, el control conductual percibido $\left(r_{s}=0.508\right)$ y las normas subjetivas $\left(r_{s}=0.361\right)$ están asociadas con la intención emprendedora de forma positiva y significativa. En cuanto al efecto, los estudiantes que desarrollaron un BP presentan los mismos niveles de actitud hacia el emprendimiento que los estudiantes que aún no lo desarrollan. Indicando que, la valoración personal positiva que tiene cada estudiante acerca de ser un emprendedor no es afectada (Ajzen, 2001; Autio, Keeley, Klofsten, Parker, \& Hay, 2001).

Resalta el hecho de que el control conductual de los estudiantes que desarrollaron un BP es mayor (mediana $=5.00$ ) al de los estudiantes que aún no lo desarrollan (mediana $=4.75$ ). La diferencia es significativa, lo que significa que desarrollar un BP aumenta el grado de percepción de la facilidad de convertirse en emprendedor (Liñán, 2004), e incrementa la confianza en las habilidades personales para tener éxito (Karali, 2013). Sin embargo, los valores de esta variable fueron los más bajos obtenidos en la escala. Dando pie a argumentar que, a pesar del efecto positivo del desarrollo del BP en el control conductual, la percepción de facilidad de convertirse en emprendedor y la confianza en las habilidades personales de los estudiantes son bajas.

En relación con las normas subjetivas, los estudiantes que desarrollaron un BP presentan los mismos niveles que los estudiantes que aún no lo desarrollan. Indicando que, el BP no afecta el HEREDIA-PORTILLO O., DELGADO-DÍAZ L. A., CORTÉS-PALACIOS E. M. 
EL EFECTO DEL DESARROLLO DE PLANES DE NEGOCIO EN LAS ACTITUDES E INTENCIONES EMPRENDEDORAS DE LOS ESTUDIANTES.

grado en que los estudiantes perciben un mayor apoyo por las personas que son importantes en su vida acerca de convertirse en emprendedor (Karali, 2013; Liñán \& Chen, 2009). De igual forma, ambos grupos presentan la misma intención emprendedora. Por lo que, el grado de convicción percibida por el estudiante de intentar establecer un negocio y que conscientemente planea hacerlo en algún momento en el futuro no es afectado (Thompson, 2009).

Además, similar a los resultados de Kolvereid \& Moen (1997), entre hombre y mujeres si hay diferencias en la actitud hacia el emprendimiento y en las normas subjetivas. Por otro lado, aunque Colombatto \& Melnik (2007) y Kolvereid \& Moen (1997) argumentan que la experiencia afecta las actitudes e intenciones emprendedoras, esta investigacion no encontró efecto alguno. La edad afecta la actitud hacia el emprendimiento e intención de iniciar un negocio, dando paso al argumento de Lévesque \& Minniti (2006), es más probable que los jóvenes inicien un negocio que los adultos. Por último, la carrera profesional afecta la intención emprendedora, algo similar a lo encontrado por Kolvereid \& Moen (1997).

De acuerdo con los resultados el desarrollo de un BP en un curso de emprendimiento no es suficiente para que los estudiantes pasen de la intención a la acción de crear un nuevo negocio (Díaz-García, Sáez-Martínez, \& Jiménez-Moreno, 2015; Rauch \& Hulsink, 2015), desarrollen competencias emprendedoras (Aldana, Ibarra, \& Lowenstein, 2011), e incrementen su deseo e interés por ser emprendedores (Tang, Chen, Li, \& Lu, 2014). Por lo tanto, no debería ser la actividad focal de aprendizaje en este tipo de educación (Colin \& Andy, 2013).

La educación emprendedora basada en BP podría fracasar en preparar a los estudiantes al no afectar la intención para dar paso a la búsqueda y descubrimiento de las oportunidades que después decidirán explotar (Lima, Nassif, Lopes, \& Silva, 2014; Blank, Engel, \& Hornthal, 2014; Shook, Priem, \& McGee, 2003). Esto hace evidente que los esfuerzos por enseñar planeación a nivel universitario podrían en realidad ser una mala asignación de recursos pedagógicos (Honig \& Samuelsson, 2011). Por lo tanto, los profesionales en educación emprendedora deben revisar el énfasis en los BPs, porque si un emprendedor no busca financiamiento, no existe otra razón para escribirlos antes de iniciar una empresa (Lange, Mollov, Pearlmutter, Singh, \& Bygrave, 2007).

Claro está, es necesario buscar más herramientas para reforzar la educación emprendedora. Por ejemplo, Lean Startup (Ries, 2011), Design Thinking (Brown, 2008), Customer Development HEREDIA-PORTILLO O., DELGADO-DÍAZ L. A., CORTÉS-PALACIOS E. M. 
EL EFECTO DEL DESARROLLO DE PLANES DE NEGOCIO EN LAS ACTITUDES E INTENCIONES EMPRENDEDORAS DE LOS ESTUDIANTES.

(Blank \& Dorf, 2012), Business Models Generation (Osterwalder \& Pigneur, 2010) o Value Proposition Design (Osterwalder, Pigneur, Smith, Bernarda, \& Papadakos, 2014), estos nuevos métodos están revolucionando la forma en que los emprendedores crean, refinan y llevan exitosamente las nuevas ideas al mercado, al mismo tiempo que reducen la incertidumbre y el riesgo a través de experimentos rápidos y baratos (Furr \& Dyer, 2014).

\section{REFERENCIAS BIBLIOGRÁFICAS}

Ahlstrom, P. \& Furr, N. (2011). Nail it then Scale it. The Entrepreneur's Guide to Creating and Managing Breakthrough Innovation. United State: NISI Institute.

Ajzen, I. (1991). The Theory of Planned Behavior. Organizational Behavior and Human Decision Processes, 50, 179-211.

Aldana, F. E., Ibarra, S. M. \& Lowenstein, R. I. (2011). El Modelo de Negocio Como Reforzador del Emprendimiento en las Universidades. Caso del Tecnológico de Monterrey Campus Ciudad de México. Revista Ciencias Estratégicas, Medellín, Colombia, 185-201.

Ashamalla, H. M., Orife, N. J. \& Abel, I. (2008). Business Plans: Are They Relevant to Venture Capitalists? Journal of Small Business and Entrepreneurship, 21(4), 381-392. https://doi.org/10.1080/08276331.2008.10593431.

Autio, E., Keeley, R. H., Klofsten, M., Parker, G. G. \& Hay, M. (2001). Entrepreneurial Intent among Students in Scandinavia and in the USA. Enterprise and Innovation Management Studies, 2(2), 145-160. https://doi.org/10.1080/14632440110094632.

Bewayo, D. E. (2015). The Overemphasis on Business Plans in Entrepreneurship Education: Why does it persist? Journal of Small Business and Entrepreneurship Development, (3), 1-7. http://dx.doi.org/10.15640/jsbed.v3n1a1.

Bhidé, A. (1999). The Origin and Evolution of New Business. Oxford: The Oxford University Press.

Blank, S. \& Dorf, B. (2012). The startup owner's manual. Pescadero: K\&S Ranch, Inc.

Blank, S., Engel, J., \& Hornthal, J. (2014). Lean LaunchPad. Evidence-Based Entrepreneurship. Educators Guide, 6th Edition. California: Venture Well.

Brinckmann, J., Grichnik, D. \& Kapsa, D. (2010). Should entrepreneurs plan or just storm the castle? A meta-analysis on contextual factors impacting the business planning-performance relationship in small firms. Journal of Business Venturing, 25, 24-40. https://doi.org/10.1016/j.jbusvent.2008.10.007.

Brown, T. (2008). Design Thinking. Harvard Business Review, 84-92. 
EL EFECTO DEL DESARROLLO DE PLANES DE NEGOCIO EN LAS ACTITUDES E INTENCIONES EMPRENDEDORAS DE LOS ESTUDIANTES.

Burke, A., Fraser, S. \& Greene, F. J. (2010). The Multiple Effects of Business Planning on New Venture Performance. Journal of Management Studies, 47(3), 391- 415. https://doi.org/10.1111/j.1467-6486.2009.00857.x.

Colin, J., \& Andy, P. (2013). Moving beyond the business plan in enterprise education. Education + Training, 55(8/9), 804-814. https://doi.org/10.1108/ET-06-2013-0077.

Colombatto, E. \& Melnik, A. (2007). Early Work Experience and the Transition into Entrepreneurship. Journal of Entrepreneurial Finance and Business Ventures, 12(1), 9-26. http://digitalcommons.pepperdine.edu/jef/vol12/iss1/3.

Cook, R. G., Belliveau, P. \& Lentz, C. (2007). The role of gender in US microenterprise business plan development. Journal of Small Business and Enterprise Development, 14(2), 241-251. https://doi.org/10.1108/14626000710746673.

Cook, R. G., Belliveau, P. \& Sandberg, M. E. (2004). Training and learning as drivers of US microenterprise business plan quality. Education + Training, 46(8/9), 398-405. https://doi.org/10.1108/00400910410569515.

Díaz-García, C., Sáez-Martínez, F. \& Jiménez-Moreno, J. (2015). Assessing the impact of the "Entrepreneurs" education programme on participants'. RUSC. Universities and Knowledge Society Journal, 12(3), 17-31. https://doi.org/10.7238/rusc.v12i3.2146.

Eschker, E., Gold, G. \& Lane, M. D. (2017). Rural entrepreneurs: what are the best indicators of their success? Journal of Small Business and Enterprise Development, 24(2), 278-296. https://doi.org/10.1108/JSBED-07-2016-0112.

Fayolle, A., \& Degeorge, J. M. (2006). Attitudes, intentions and behaviour: new approaches to evaluating entrepreneurship education. En A. Fayolle, \& H. Klandt, International Entrepreneurship Education. Issues and Newness (74-92). UK: Edward Elgar Publishing Limited.

Fayolle, A., \& Liñán, F. (2014). The future of research on entrepreneurial intentions. Journal of Business Research, 67, 663-666. https://doi.org/10.1016/j.jbusres.2013.11.024.

Furr, N. (2012). Burn Your Business Plan! Obtenido de Forbes: https://www.forbes.com/sites/nathanfurr/2012/02/24/burn-your-businessplan/\#53f921d176cd

Furr, N., \& Dyer, J. (2014). The innovator's method: bringing the lean startup into your organization. United State of America: Harvard Business School Publishing.

García Cabrero, B. (2009). Manual de métodos de investigación para las ciencias sociales: un enfoque basado en proyectos. México: Editorial El Manual Moderno.

Hernández, S. R., Fernández, C. C. \& Baptista, L. M. (2014). Metodología de la investigación. 6a Edición. México: McGraw-Hill/ Interamericana Editores, S.A. de C.V.

Hinton, P. R., Brownlow, C., McMurray, I. \& Cozens, B. (2004). SPSS Explained. New York: Routledge. 
EL EFECTO DEL DESARROLLO DE PLANES DE NEGOCIO EN LAS ACTITUDES E INTENCIONES EMPRENDEDORAS DE LOS ESTUDIANTES.

Honig, B. (2004). Entrepreneurship Education: Toward a Model of Contingency-Based Business Planning. Academy of Management Learning and Education, (3), 258-273. https://doi.org/10.5465/amle.2004.14242112.

Honig, B. (2012). Planning for Entrepreneurial Finance and Capital: a Critical Review of the Importance of Teaching Business Planning. En D. Cumming, The Oxford Handbook of Entreprenerial Finance (229-254). New York: Oxford University Press.

Hormozi, A. M., Sutton, G. S., McMinn, R. D., \& Lucio, W. (2002). Business plans for new or small businesses: paving the path to success. Management Decision, 40(8), 755-763. https://doi.org/10.1108/00251740210437725.

Hussain, A. (2015). Impact of Entrepreneurial Education on Entrepreneurial Intentions of Pakistani Students. Journal of Entrepreneurship and Business Innovation. Macrothink Institute, 2(1). 43-53.

Karali, S. (2013). Tesis maestría "The Impact of entrepreneurship education programs on entrepreneurial intentions: An application of the theory of planned behavior". Obtenido de Erasmus University Rotterdam: http://thesis.eur.nl/pub/13460/MA-Thesis-Sofia-Karali357726\%20.pdf.

Karlsson, T. (2005). JIBS Dissertation Series No. 330. Business Plan in New Ventures. An Institutional Perspective. Jönköping: Jönköping International Business School.

Katz, J. A. (2003). The chronology and intellectual trajectory of American entrepreneurship education 1876-1999. Journal of Business Venturing, 18, 283-300. https://doi.org/10.1016/S0883-9026(02)00098-8.

Kirby, D. A. (2004). Entrepreneurship education: can business schools meet the challenge? Education + Training, 46(8/9), 510-519. https://doi.org/10.1108/00400910410569632.

Kolvereid, L. \& Moen, Ø. (1997). Entrepreneurship among business graduates: does a major in entrepreneurship make a difference? Journal of European Industrial Training, 21(4), 154160. http://dx.doi.org/10.1108/03090599710171404.

Krueger, F. N. \& Reilly, D. M. (2000). Competing Models of Entrepreneurial Intentions. Journal of Business Venturing, 15, 411-432. https://doi.org/10.1016/S0883-9026(98)00033-0.

Krueger, N. F. \& Carsrud, A. L. (1993). Entrepreneurial intentions: Applying the theory of the planned behaviour. Entrepreneurship \& Regional Development, 5, 315-330. https://doi.org/10.1080/08985629300000020.

Kuratko, F. D. (2005). The Emergence of Entrepreneurship Education: Development, Trends, and Challenges. 1042-2587. Entrepreneurship Theory and Practice, 29(5), 577-597. https://doi.org/10.1111/j.1540-6520.2005.00099.x.

Lange, E. J., Mollov, A., Pearlmutter, M., Singh, S. \& Bygrave, D. W. (2007). Pre-start-up Formal Business Plans and Post-start-up Performance: A Study of 116 New Ventures. Venture Capital, 9(4), 237-256. https://doi.org/10.1080/13691060701414840. 
EL EFECTO DEL DESARROLLO DE PLANES DE NEGOCIO EN LAS ACTITUDES E INTENCIONES EMPRENDEDORAS DE LOS ESTUDIANTES.

Lange, J. E., Marram, E., Jawahar, A. S., Yong, W. \& Bygrave, W. (2011). Does an entrepreneurship education have lasting value? a study of careers of 4,000 alumni. Frontiers of Entrepreneurship Research, 31(6), 210-224.: http://digitalknowledge.babson.edu/fer/vol31/iss6/2.

Lévesque, M. \& Minniti, M. (2006). The effect of aging on entrepreneurial behavior. Journal of Business Venturing, 26, 177-194. https://doi.org/10.1016/j.jbusvent.2005.04.003.

Levin, R. I. \& Rubin, D. S. (2004). Estadística para Administración y Economía. México: Pearson Educación.

Lima, E., Nassif, V. M., Lopes, R. M. \& Silva, D. (2014). Entrepreneurship Higher Education and Students' Entrepreneurial Intentions in Brazil - Report on the Brazilian GUESSS 2013-2014. Grupo APOE - Grupo de Estudo sobre Administração de Pequenas Organizações e Empreendedorismo, PPGA-UNINOVE. WP n. 2014-05. Obtenido de Global University Entrepreneurial Spirit Student's Survey http://www.guesssurvey.org/publications/publications/national-reports.html

Liñán, F. (2004). Intention-Based Models of Entrepreneurship Education. Obtenido de Research Gate: https://www.researchgate.net/publication/235937886_IntentionBased_Models_of_Entrepreneurship_Education

Liñán, F. \& Chen, Y. W. (2009). Development and Cross-Cultural Application of a Specific Instrument to Measure Entrepreneurial Intentions. Entrepreneurship Theory and Practice, 593-617. https://doi.org/10.1111/j.1540-6520.2009.00318.x.

Longenecker, J. G., Moore, C. W., Petty, J. W. \& Palich, L. E. (2010). Administración de pequeñas empresas.Lanzamiento y crecimiento de iniciativas emprendedoras, (14a. ed.) México: Cengage Learning Editores, S.A.

Morris, M. G. \& Venkatesh, V. (2000). Age differences in technology adoption decisions: Implications for a changing work force. Personnel Psychology, 53(2), 375- 403.

Osterwalder, A. \& Pigneur, Y. (2010). Business Model Generation. Hoboken: John Wiley \& Sons, Inc.

Osterwalder, A. Pigneur, Y., Smith, A., Bernarda, G. \& Papadakos, P. (2014). Value Proposition Design. Hoboken: John Wiley \& Sons, Inc.

Qureshi, M. S. \& Mahdi, F. (2014). Impact of Effectuation Based Intervations on the Intention to Start a Business. Business Review, 9(2), 143-157.

Rauch, A. \& Hulsink, W. (2015). Putting Entrepreneurship Education Where the Intention to Act Lies: An Investigation Into the Impact of Entrepreneurship Education on Entrepreneurial Behavior. Academy of Management Learning \& Education, 14(2), 187-204. http://dx.doi.org/10.5465/amle.2012.0293.

Ries, E. (2011). The Lean Startup. How today's Entrepreneurs Use Continuous Innovation to Create Radically Successful Businesses. United State: Crown Business. 
EL EFECTO DEL DESARROLLO DE PLANES DE NEGOCIO EN LAS ACTITUDES E INTENCIONES EMPRENDEDORAS DE LOS ESTUDIANTES.

Shook, C. L., Priem, R. L. \& McGee, J. E. (2003). Venture Creation and the Enterprising Individual: A Review and Synthesis. Journal of Management, 29(3), 379-399. https://doi.org/10.1016/S0149-2063_03_00016-3.

Solomon, G. (2007). An examination of entrepreneurship education in the United States. Journal of Small Business and Enterprise Development, 14(2), 168-182. https://doi.org/10.1108/14626000710746637.

Tamayo y Tamayo, M. (2012). El proceso de la investigación científica: incluye evaluación y administración de proyectos de investigación. México: Limusa.

Tang, M., Chen, X., Li, Q., \& Lu, Y. (2014). Does Chinese university entrepreneurship education fit students' needs? Journal of Entrepreneurship in Emerging Economies, 6(2), 163-178. https://doi.org/10.1108/JEEE-02-2014-0002.

Thompson, E. R. (2009). Individual Entrepreneurial Intent: Construct Clarification and Development of an Internationally Reliable Metric. Entrepreneurship Theory and Practice, 33(3), 669- 694. https://doi.org/10.1111/j.1540-6520.2009.00321.x.

Tung, C. L. (2011). Tesis doctoral: The impact of Entrepreneurship Education on Entrepreneurial Intention of Engineering Students. Obtenido de University of Hong Kong: http://lbms03.cityu.edu.hk/theses/c_ftt/phd-meem-b40869337f.pdf

UDG. (25 de marzo de 2016). Sistema Integral de Información y Administración Universitaria. Recuperado el 09 de marzo de 2016, de Universidad de Guadalajara: http://www.siiau.udg.mx/

UDG. (25 de marzo de 2016a). Programas de licenciatura. Desarrollo de Emprendedores. Obtenido de Centro Universitario de Ciencias Económico Administrativas. Universidad de Guadalajara: http://www.cucea.udg.mx/es/consulta-programas-de-asignatura

Vincett, P. S. \& Farlow, S. (2008). "Start-a-Business": an experiment in education through entrepreneurship. Journal of Small Business and Enterprise Development, 15(2), 274-288. https://doi.org/10.1108/14626000810871673.

Wong, K. P., Ho, P. Y. \& Low, C. P. (2014). Do University Entrepreneurship Programs Influence Students' Entrepreneurial Behavior? An Empirical Analysis of University Students in Singapore. En S. Hoskinson, \& D. F. Kuratko, Innovative Pathways for University Entrepreneurship in the 21st Century (Advances in the Study of Entrepreneurship, Innovation \& amp; Economic Growth, 24, 65-87. http://dx.doi.org/10.1108/S1048473620140000024003). United State: Emerald Group Publishing Limited. 